\title{
HISTORIA DE LA FAMILIA COMO HISTORIA ECONÓMICA: TEMAS Y FUENTES PARA LIMA EN EL SIGLO XIX
}

\author{
Christine Hunefeldt*
}

$\mathrm{E}$

n la década de los setenta del presente siglo algunos historiadores latinoamericanos unieron esfuerzos para crear los lineamientos para el estudio de la historia económica en América Latina. Muchos de ellos eran recientes egresados de universidades en Francia y estaban imbuidos de los alcances de la escuela francesa. En un esfuerzo para entonces notorio, intentaron unir lo aprendido en Europa con las peripecias de las experiencias coloniales latinoamericanas, para construir una agenda de investigación. Como ejes de su propuesta se mencionaron la necesidad de generar estudios sobre precios, balanzas comerciales, la reconstrucción del producto interno bruto, las repercusiones de las exportaciones sobre la dinámica económica interna, el mercado interno, las causas y las consecuencias de los ciclos ascendentes y descendentes. A ello se agregaba una preocupación por la componente humana, entendida fundamentalmente como elemento demográfico y como fuerza de trabajo. Esta agenda representaba una versión "más científica" de lo que hasta entonces -en América Latina- se había publicado como capítulos de la historia económica.

Gran parte de esta agenda inicial no llegó a concretarse, en parte porque muy pronto dejó de ser una agenda que continuara siendo pensada como latinoamericana. ${ }^{1}$ Desde entonces, la agenda se ha multiplicado y dispersado, sin nuevos horizontes de sistematización (Halperin), siguiendo un desarrollo que más allá de América Latina responde también a la fragmentación y desorientación más generalizada de los temas históricos y la historia misma.

\footnotetext{
* Profesora del Departamento de Historia en la Universidad de California, La Jolla.

' Sólo en los últimos tiempos se han retomado algunos esfuerzos comparativos, significativamente en función de los temas que bordean las crisis económicas del siglo xx, y son comparaciones encaradas sobre todo por economistas y no por historiadores.
} 
Shane Hunt, también en los setenta, elaboró una agenda de historia económica específica para el caso peruano, y fue él quien por primera vez de manera sistemática indicó fuentes para encarar algunas tareas. No estoy muy segura de si a esta propuesta habría mucho más que agregar. Las dudas provienen de la definición de lo que es la historia económica. Hemos visto en los últimos veinte años cómo la historia económica "pura" de los setenta ha ido acercándose cada vez más a la historia social, o como lo preferiría Hobsbawm, a la historia de la sociedad. Este parece un desarrollo lógico para sociedades como las latinoamericanas, con una gran diversidad y heterogeneidad cultural y racial.

De manera más concreta la ampliación de temas en el campo de la historia económica también tiene que ver con el hecho de que muchas de las soluciones fueron propuestas y llevadas a cabo con criterios que siguen exclusivamente las lógicas de la teoría económica, tendiendo así a dejar de lado los componentes humanos; lo que ha significado una secuela de fracasos, en cuanto que los objetivos de desarrollo no han sido alcanzados. Todo lo contrario, las crisis son más profundas, la gente se alimenta peor y está menos contenta. Fracasos reiterados - sobre todo, en las experiencias más recientes de la década de los 80 - exigen alternativas de política económica más englobantes. No sorprende en este contexto, encontrar llamados a la imaginación y la creatividad en una bibliografía que frenéticamente cuestiona las bases mismas del análisis histórico. Los fracasos, las dudas y el reconocimiento de la diversidad han iniciado e intensificado la búsqueda de alternativas. Las preguntas que el historiador económico se plantea (o debería plantearse) se han ampliado. Así, resulta más complejo delimitar el campo de la historia económica y, concomitantemente, exige repensar el uso de las fuentes conocidas e incluir fuentes nuevas.

A pesar de la dispersión, empero, tal vez sea posible reunir a los nuevos temas de la historia económica en dos grandes áreas o perspectivas: la dinámica de las relaciones de género, la cuestión acerca de la reproducción biológica y social que parte de los crecientes aportes de una literatura feminista, y la internacionalización de las relaciones económicas y sociales. Esta internacionalización es una lógica dictaminada por el capital. La multinacionalización del capital ha avanzado mucho más rápidamente que su correlativa organización social, el movimiento obrero internacional o la organización de los marginados del mismo proceso. Muchas de las negociaciones sobre condiciones laborales no se dan más en el diálogo entre obreros, empresas locales y Estado nacional, sino que están intermediadas por gobiernos externos, movimientos obreros externos y organizaciones internacionales. Para los países de América Latina ésta es una experiencia mucho más vieja que para los países de Europa occidental, Japón y Estados Unidos. El proceso de inserción al mercado internacional y de industrialización estuvo precedido por el diálogo (o si se quiere, el conflicto) extranacional. Esta aseveración tan obvia para muchos, encierra, sin embargo, algunas preguntas que implican nuevas metodologías y nuevas 
respuestas. ¿Cuáles fueron los canales de participación construidos por los obreros y los marginados en la definición de la presencia del capital? ¿Cómo interactuaron Estado nacional, burguesía internacional y obreros? ¿Hasta qué punto el diálogo entre capital internacional y obreros debilitó la formación de Estados nacionales? ¿Qué significan estos procesos en cuanto a la construcción de identidades colectivas y de clase? ¿Qué implicaciones tienen estos procesos sobre la construcción (o desarticulación) de la vida de comunidad, de la vida familiar?

Así, la dinámica de las relaciones de género y de la reproducción biológica y social son temas que están reacomodando el ámbito de la historia económica hacia polos distintos, pero a la vez complementarios. El primero hacia una comprensión de los fenómenos sociales a nivel micro, el segundo hacia una ampliación del nivel macro, que tiende a desencajar los confines del Estado-nación como unidad analítica viable. El énfasis sobre estudios históricos regionales es una consecuencia de esto último.

Todavía estamos en la antesala de un matrimonio feliz de la unidad doméstica y la economía-mundo, a pesar de los recientes esfuerzos por recrear históricamente a la "comunidad" como eslabón esencial para pensar en un mundo distinto, es decir, opuesto a la vigencia última de la socialdemocracia. En esta perspectiva la "cultura" deja de ser el reservorio de todo lo que la economía no puede explicar.

Los avances aún incipientes logrados a partir de una "perspectiva de género", han hecho más compleja la definición de la historia económica. La producción, la distribución y el consumo (síntesis de los temas clásicos de la economía) han sido ampliados con el reconocimiento de una dinámica de reproducción que todavía divide a las sociedades entre hombres y mujeres. Paulatinamente se ha llegado a reconocer conscientemente que una parte importante de la reproducción de la sociedad depende del accionar de las mujeres (y hombres), cuyas vidas transcurrieron y transcurren en la esfera doméstica, en las relaciones de barrio, en la vida interior de la comunidad mayor. En otras palabras, hay instancias de la reproducción de la vida cotidiana que han estado alejadas de la apreciación histórica, y que con el cambio de acento en los paradigmas pensados adquieren un peso propio. Este ámbito de preguntas, más orientado a las relaciones sociales y al universo socio-cultural, está siendo descubierto en su vertiente económica e iluminando las esferas más recónditas del uso que hacen diversos sistemas de producción, esencialmente el capitalismo, de la energía humana. Paralelamente estamos comenzando a entender mejor también cuáles son los mecanismos de resistencia generados en la esfera doméstica que se oponen (o usan selectivamente) a las exigencias del mercado. En contraste con las propuestas de la "articulación de los modos de producción" que reconocía la utilización y la resistencia de formas más "tradicionales" de captación de recursos para el sistema de producción dominante, la perspectiva 
de género sitúa el eje analítico en la familia, en la comunidad, y no en las imperfecciones necesarias de la cristalización de un modo de producción capitalista. El lente del historiador está puesto en otro universo de relaciones sociales.

Para América Latina, esta nueva perspectiva es doblemente relevante. Una creciente bibliografía sobre el tema evidencia de manera cada vez más clara que la familia (y su expresión más englobante, la comunidad) es la mínima base de organización social y económica frente a gremios débiles, sindicatos débiles, Estados débiles. No es que en los países desarrollados la familia no sea importante. Sí lo es, pero su rol socioeconómico es distinto. En América Latina diferentes tipos de familia son parte esencial de los principios organizativos de la sociedad en su conjunto. En el interior de las familias las mujeres juegan un rol central. En un tono más contemporáneo y político, desde las madres de la plaza de mayo hasta las bartolas, la gran cantidad de mujeres afiliadas a Sendero Luminoso y el sello político de los vasos de leche; todo indica que desde la perspectiva de género es necesario reevaluar nuestras aproximaciones históricas. Ello supone convertir a la perspectiva 'micro' en un tema macro, y plantear nuevas preguntas: ¿en qué momentos y por qué la familia representó la unidad económica más importante? ¿Qué tipo de estrategias económicas fueron ideadas por la familia para acomodarse o protestar contra situaciones de crisis? ¿Qué rol jugaron las mujeres en las cambiantes situaciones familiares? ¿Cómo se formularon en la unidad doméstica las decisiones de consumo? Y, en términos más políticos, ¿cómo se formularon, desde las experiencias en el seno de lo doméstico, reivindicaciones con tonos feministas o proletarias o burguesas?

Es probable que el tema que más se aproxima a la perspectiva de género y la internacionalización de las relaciones económicosociales, sea de la construcción de identidades. Si bien desde plataformas muy dispares, su conjunción permite pensar la historia económica desde perspectivas tendencialmente prometedoras y menos esquemáticas. Aun si, como presumo, "la construcción de identidades", parecerá un tema muy alejado de lo que se suele definir como historia económica, la identidad es un resorte para la acción que sintetiza muchas experiencias más obviamente económicas.

Es, pretenciosamente, sobre este conjunto de lineamientos que quisiera sugerir que la historia de la familia es también una cara de la historia económica. Aun más, una cara que urgentemente requiere de una fisonomía propia y precisa. Al final de una investigación (o de varias), siempre estamos en mejores condiciones de responder a la pregunta: ¿y por qué las cuestiones que uno se plantea son importantes? Retrospectivamente - porque los cometidos nunca son tan claros al inicio- diría que la justificación de la elección de temas que giran en torno a la historia de la familia tiene dos ejes: 1) destacar aquellos elementos de la vida cotidiana que la historia económica ha dejado de lado para convertir nuestra comprensión de las sociedades en algo más completamente construido, 
y 2) visualizar el potencial político que se derivaría de un reconocimiento histórico de los principios organizativos de las sociedades latinoamericanas, siendo uno de los principios importantes la familia, y dentro de ella, la mujer.

Ya Hunt en su balance sobre temas y fuentes postulaba que más allá de los temas clásicos de la historia económica, elementos como la educación (una historia de la educación desde un punto de vista económico) y los gastos familiares (en tanto orientación de los ahorros por parte de las elites) eran medulares para entender los obstáculos al crecimiento. Su definición ahora puede y debe ser ampliada. Uno de los obstáculos de la ampliación temática sobre estos lineamientos es que los aportes y resistencias a la reproducción de la vida cotidiana son difíciles de medir, ${ }^{2}$ ya que no pueden ser objeto de regresiones y estandarización estadística, como eventualmente las balanzas comerciales.

La investigación que aquí queremos comentar se inició diez años atrás, y gira en torno a las familias limeñas entre fines del periodo colonial e inicios del siglo XX, un "largo siglo XIX", que todavía es el paria de la historia latinoamericana, frente a los acentos puestos sobre el periodo colonial y el siglo XX. A pesar de esfuerzos recientes (véase bibliografía) que reconstruyen temas del siglo XIX más allá de la afirmación de que fue el siglo en que se reordenaron las coordenadas de inserción al mercado internacional, aun hoy -ese largo siglo de reconformación liberal, burguesa, moderna (o como quiera llamársele)- es una gran incógnita. Particularmente en Perú el tema y la visión que aquí se proponen son prácticamente vírgenes. Creemos que la perspectiva de género (reproducción) y de la historia de la familia, aparte de su pertinencia intrínseca, permite encarar los grandes cambios (capitalistas, industriales, liberales) atribuidos al siglo XIX desde sus efectos e impulsos sobre y desde la vida de la familia.

En los últimos diez años hemos avanzado mucho en nuestra comprensión sobre el papel de la familia en diferentes coyunturas económicas y espacios regionales, aun cuando el análisis ha estado centrado en las elites. Y, es que, como señala Hamerow: "There is a compelling logic which insists that the policies and decisions of masters of politics or captains of industry affect society's vital interests more profoundly than the folkways and mores of hewers of wood and drawers of water." Lo que sabemos sobre las familias de las elites es más para Buenos Aires, que para Argentina, más para ciudad de México que para México, más para Sao Paulo y Río que para Brasil, y más para México, Argentina y Brasil que para Perú. Ello denota una clara disparidad de los avances y un acento sobre el contexto urbano, lo que a su vez dificulta un análisis

2 En la misma dirección, la historia económica aún es muy pobre en el manejo de sus instrumentos cuando se trata de analizar los comportamientos económicos de otros agentes sociales que tienden a escapar de las redes del mercado: los campesinos y los sectores informales. 
comparativo (y explica por qué el marco comparativo es Europa y Estados Unidos), y hace imposible medir los cambios dispares incluso en el interior de una misma región o país. Están abiertas las preguntas en torno a las capas medias y populares, tanto en el ámbito urbano como rural. Respuestas para capas medias y populares, desde la perspectiva familiar, son la prolongación analítica necesaria de la "historia desde abajo".

En Perú existen archivos con marcados desniveles en cuanto a la organización y catalogación de sus materiales. Hay muchos archivos que no pasan de ser perdidos cúmulos de papeles en algún cuartucho o traspatio, cuando milagrosamente se han salvado de las regulares limpias a que son sometidos los "papeles viejos". La transición del "papel viejo" a "documento histórico" responde a la incansable labor del Archivo General de la Nación, a cuyo cargo están los archivos departamentales. No todos los departamentos del país (y tampoco las nuevas regiones), sin embargo, cuentan con archivos departamentales. Su fundación y construcción ha sido tarea de los últimos quince años. En algunos casos (Piura, por ejemplo) la catalogación y la detección de fuentes ha progresado muchísimo. En otros (Puno, por ejemplo) el archivo no cuenta aún ni siquiera con un local adecuado.

Para cualquier investigación sobre familia, el Archivo Arzobispal probablemente contiene las series más importantes y de consulta obligada.

Para información complementaria, la Biblioteca Nacional (sala de investigaciones) es de suma importancia, así como su hemeroteca. El Archivo Municipal provee de algunos datos demográficos sobre la evolución limeña, e información acerca de lo que es el ámbito de la asistencia social está concentrada en el Archivo de la Beneficencia Pública.

Nuestro análisis, por ahora, se centra en las familias limeñas, pero incluye las experiencias de algunas familias rurales, en gran medida porque Lima misma durante el siglo XIX y más tarde, fue una sociedad con un altísimo grado de movilidad física. Alrededor de 35\% de los habitantes limeños provenían de zonas rurales, de provincias, $15 \%$ eran migrantes extranjeros (fundamentalmente europeos y procedentes de otras naciones sudamericanas), y el resto había nacido en Lima en cualquier momento del siglo.

Para un periodo en el que prácticamente no existen censos, un análisis de la familia hace necesaria la reconstrucción de algunos indicadores demográficos básicos. Los únicos censos nacionales existentes para Perú son el de 1792 y el de 1876. El que le sigue es de 1940 . Un crecientemente sofisticado análisis demográfico a partir de los registros parroquiales puede ampliar nuestros conocimientos sobre la cantidad de personas, sus patrones migracionales y el ciclo de vida. Éste es un trabajo que para el siglo XXX peruano casi no ha empezado. Sin embargo, por ahora, hay algunos atajos posibles. Las licencias matrimoniales (almacenadas en el Archivo Arzobispal) y los testamentos contenidos en los protocolos notariales (Archivo General de la Nación) permiten reconstruir los patrones de movilidad 
geográfica y social. Las licencias matrimoniales registran la edad de los cónyuges (más sistemáticamente para mujeres que para hombres), ocasionalmente su pertenencia racial, su procedencia e indican el grado de familiaridad de los contrayentes con sus testigos. Estos datos son los que permiten reconstruir los patrones de movilidad de la sociedad limeña, los cambios en la edad matrimonial -a su vez un indicador de las condiciones económicas de los diferentes grupos raciales y sociales-, y el grado de endogamia racial, geográfica y económica. Los testamentos, permiten verificar la confiabilidad de los datos sobre migración (en tanto todo testante indica el lugar de su nacimiento), sobre edad matrimonial, y permiten tener una idea sustantivamente mejor sobre la ubicación social de los testantes a partir de una evaluación de la composición de bienes y deudas enunciadas en los testamentos.

Una posibilidad adicional que ofrecen los testamentos es la reconstrucción familiar. Si se logra armar un muestra representativa por grupo social a lo largo de dos o tres generaciones, es posible observar los patrones de movilidad social por ocupación, y con un poco de imaginación, por clase social. Lograr esto último en un tiempo prudencial depende mucho del ordenamiento y la catalogación de los archivos consultados. Un índice alfabético de todos los testantes en los protocolos notariales de Lima ha ayudado notoriamente a este proyecto.

Otro tema demográfico que puede ser abordado desde las licencias matrimoniales y los testamentos es el de la legitimidad. En las licencias casi siempre se indica si los contrayentes son ellos mismos hijos legítimos, ilegítimos o naturales. Lo mismo es cierto para los testamentos, que tienen una ventaja adicional, y es que el testante enumera la cantidad de hijos procreados, así como la cantidad de hijos muertos. A veces incluso se indica a qué edad murieron. Casi sin excepción la obligación moral en aras del Último Juicio, hizo que los testantes declararan, asimismo, si durante su vida habían procreado hijos ilegítimos o naturales. De esta manera es posible, aproximarnos a algunos índices sobre mortalidad, fertilidad y natalidad (que tal vez nos ayuden a explicar el lento crecimiento de Lima, frente a otras ciudades latinoamericanas durante este siglo). Estos, a su vez son datos indispensables para evaluar los patrones de herencia (también averiguables a partir de los testamentos), la importancia de los albaceazgos (muchas veces representando la interferencia de la familia ampliada), y el control sobre bienes ejercidos por las mujeres (sobre todo las viudas). Un dato adicional contenido en los testamentos se refiere a dotes y gananciales, y la cambiante interrelación entre ambas. Preguntas como por qué los mercaderes y agricultores fueron los últimos en abandonar las dotes definidas como una transferencia del padre de la novia al marido de ésta, pueden ser respondidas a partir de una sistematización de esta información. ${ }^{3}$

${ }^{3}$ Para el proyecto sobre familias en Lima, se ha recurrido a un vaciado completo de los testamentos para cada diez años, lo cual hace un total aproximado de 1800 testamentos en los programas kedit/spss. 
En oposición a lo que generalmente se piensa, los testamentos no tienen sesgos dramáticos hacia los hombres y hacia las capas altas de la sociedad limeña. Existen, para el siglo XIX, casi igual cantidad de hombres y mujeres que redactan testamentos y, si se evalúa la composición de los bienes, los testantes reflejan bastante bien las disparidades y la heterogeneidad económica de los habitantes limeños. Es por ello, que pienso que los testamentos -aparte del valor que tienen para la reconstrucción de datos demográficos- son tal vez la fuente más adecuada para intentar una aproximación a las bases económicas de la vida familiar y los cambios en el tiempo.

Poesía, cantos y folclore, así como arquitectura, pintura y escultura, y los comentarios de viajeros y observadores contemporáneos, han sido las fuentes más obvias para la reconstrucción de la vida popular. Pero hay otro grupo de documentos que permite enriquecer la información sobre la vida familiar (popular y no popular): los juicios civiles y criminales, tanto en el juzgado eclesiástico como en los fueros civiles. Los expedientes judiciales son una fuente inagotable de información sobre la vida cotidiana familiar y las características del conflicto familiar. En algunos casos excepcionales, los diarios complementan la riqueza de la información anterior. A partir de estas fuentes es posible reconstruir canastas de consumo, niveles de ingreso, utilización de los recursos, capacidad de acumulación, la toma de decisiones sobre el consumo y la inversión del ingreso, así como lo que Wallerstein denominaba las características cambiantes del income pooling. Todas estas fuentes no están sujetas a limitaciones temporales. Existen desde el siglo XVI hasta el siglo XX en los archivos nacionales y departamentales, si bien su búsqueda, por falta de catalogación, puede ser azarosa. ${ }^{4}$

Particularmente ricos son los expedientes en el Archivo Arzobispal de Lima, titulados Litigios matrimoniales, Causas de divorcio y Expedientes de nulidad, que representan tres estadios diferentes de gravedad de los conflictos matrimoniales. Mientras que testamentos y licencias matrimoniales permiten reconstruir básicamente los lineamientos, de la vida "normal", los expedientes en el Archivo Arzobispal subrayan las dimensiones conflictivas de la vida cotidiana. Y es a través del conflicto que se cristalizan expectativas cambiantes de la vida y la articulación familiar, las cambiantes opiniones sobre los hijos y la injerencia de suegros y suegras y de vecinos. Los "litigios matrimoniales" son, por lo general, quejas menores y pasajeras presentadas por hombres y mujeres sobre el comportamiento del otro cónyuge. La "causa de divorcio" es algo más seria, en tanto,

${ }^{4}$ Desmintiendo la falta de información para el siglo XxX, este tipo de información es particularmente rica para el mencionado siglo, y promete una comprensión mucho más sofisticada del proceso histórico de este siglo. Lo que personalmente opté por hacer frente a esa masa de metros lineales de documentación es trabajar bajo la lógica del muestreo, que simplemente significó tomar diez legajos cada cinco años a lo largo del siglo XIX de cada una de las series (civiles y criminales). 
se pide la separación temporal de los cónyuges y mayormente requiere de la presentación de muchos testigos. Los "expedientes de nulidad" apuntan a la cancelación del vínculo matrimonial siguiendo algunas de las causales de nulidad reconocidas por el derecho canónico. En no pocos casos, una pareja presentó a lo largo de muchos años de vida conyugal un juicio en las tres instancias. Cuando esto es el caso, es posible a veces reconstruir décadas de conflicto y observar la evolución del mismo, tanto a través de la cambiante relación entre hombres y mujeres, como a través del cambiante contexto socioeconómico. Con suerte, la misma pareja siguió un expediente en el fuero civil (por ejemplo, sobre división de bienes o asignación de alimentos para hijos o mujer u hombre). Ello permite contrastar la evolución del conflicto con sus efectos sobre la vida material de la familia. La condición óptima es cuando luego de ubicar un litigio matrimonial, una causa de divorcio, un pedido de nulidad, y uno o varios expedientes civiles y criminales, para el mismo caso, se logra detectar los testamentos de los litigantes. Con ello, todo el ciclo de vida familiar queda representado en los documentos y permite medir las implicaciones del conflicto sobre la acumulación familiar, su inserción en el ámbito de las relaciones barriales, y evaluar las cambiantes percepciones sobre el rol de la familia.

La combinación de muchas fuentes en diferentes repertorios, ordenadas por "casos de familias" (unos 2000 , sobre una población entre 50000 en 1800 y 110000 en 1900), proveen de una idea muy clara sobre la importancia de la familia en la construcción del siglo XIX desde la perspectiva de la familia. Desde los cambios en la estructura de la familia, la injerencia de la familia ampliada, hasta la reconstrucción de las relaciones de género y las reivindicaciones femeninas desde el ámbito de las dotes y las gananciales, estas series sugieren un patrón de evolución social muy distinto a aquel registrado para otras latitudes. Tomo esta última aseveración como prueba de la utilidad de la perspectiva propuesta y como pretexto para invitar a la lectura y a la crítica del libro que con algo de suerte estará en el mercado el año próximo.

\section{BIBLIOGRAFÍA}

Incluye algunas publicaciones sobre la historia económica del Perú del siglo XIX publicadas después de 1986.

BlanCHARD, PETER, Slavery and abolition in early Republican Peru, Scholarly Resources Inc., Wilmington, 1992.

Bonilla, HeraClio (comp.), Las crisis económicas en la historia del Perú, ClAHES/Fundación Ebert, Lima, 1986.

Contreras, Carlos, Mineros y campesinos en los Andes. Mercado laboral y economía campesina en la sierra central. Siglo XIX, IEP, Lima, 1987. 


\section{Christine Hunefeldt}

CORTÉs Conde, RoBerto y StANLEy J. STEIN (comps.), Latin America: a guide to economic history, 1830-1930, University of California Press, Berkeley, Los Ángeles, Londres, 1977.

GoOtenberg, PAul, Between silver to guano. Commercial policy and the State in postindependence Peru, Princeton University Press, Princeton, N. J., 1989.

HALPERIN, Tulio, "Para un balance del estado actual de los estudios de Historia Latinoamericana", en HISLA, vol. v, ler. semestre (reproducido también en Christopher Mitchell (comp.), Changing perspectives in Latin American studies. Insights from six disciplines, Stanford University Press, Stanford, 1988, pp. 13-62, bajo el título "The State of Latin American history").

HAMEROW, THEODORE S., Reflections on history and historians, The University of Wisconsin Press, Madison, particularmente pp. 162-204, 1987.

HUNEFELDT, CHRISTINE, "Viejos y nuevos temas de la historia económica del siglo XIX", en H. Bonilla (comp.), op. cit.

HuNT, SHANE, "Some tasks in peruvian economic history, 1830-1930", Woodrow Wilson School, Princeton, discussion paper \# 25.

MANRIQUE, NELSON, Mercado interno y región. La sierra central 1820-1930, DESCO, Lima, 1987.

QUIROZ, ALFONSO W., Banqueros en conflicto. Estructura financiera y economía peruana, 1884-1930, CIUP, Lima, 1989. 